\title{
Design for a portable calibration system for the Full-sun UV Rocket SpecTrometer instrument
}

\author{
Genevieve D. Vigil $\odot,{ }^{\mathrm{a}, *}$ Amy Winebarger, ${ }^{\mathrm{b}}$ Laurel Rachmeler, ${ }^{\mathrm{b}, \mathrm{c}}$ \\ Nicolas Donders $\odot,{ }^{d}$ P.S. Athiray $\odot,{ }^{\text {a }}$ Ken Kobayashi, ${ }^{\text {b }}$ \\ and Charles Kankelborg ${ }^{\mathrm{e}}$ \\ ${ }^{a}$ University Space Research Association, NASA Marshall Space Flight Center, Huntsville \\ Alabama, United States \\ ${ }^{b}$ NASA Marshall Space Flight Center, Huntsville, Alabama, United States \\ ${ }^{\mathrm{c}}$ NOAA National Centers for Environmental Information, Boulder, Colorado, \\ United States \\ ${ }^{\mathrm{d}}$ University of Alabama Huntsville, Huntsville, Alabama, United States \\ ${ }^{\mathrm{e}}$ Montana State University, Bozeman, Montana, United States
}

\begin{abstract}
The Full-sun Ultraviolet Rocket SpecTrograph (FURST) is a sounding rocket designed to acquire the first full-disk integrated high resolution vacuum ultraviolet (VUV) spectra of the Sun. The data enable analysis of the Sun comparable to stellar spectra measured by astronomical instruments such as those on board the Hubble Space Telescope. The mission is jointly operated by teams at Montana State University (MSU), developing the instrument, and Marshall Space Flight Center (MSFC), developing the camera and calibration systems, and is scheduled to launch from White Sands Missile Range, New Mexico, in 2022. This mission requires the development of a pre- and post-launch calibration plan for absolute radiometric and wavelength calibration to reliably generate Hubble analogue spectra. Absolute radiometric calibration, though initially planned to be performed at the National Institute for Standards and Technology (NIST) calibration facilities, is now planned to be completed with a portable VUV calibration system provided by MSFC, due to instrument incompatibilities with NIST infrastructure. The portable calibration system is developed to provide absolute wavelength calibration and track changes in calibration over the duration of the mission. The portable calibration system is composed mainly of a VUV collimator equipped with an extreme ultraviolet line source and calibrated photodiodes. The calibration system is developed to accommodate both repeatable wavelength and radiometric testing of the FURST instrument at various test sites before and after launch. Presented here are the requirements, design, and implementation of this portable calibration system with a focus on those features most significant to radiometric measurements. () 2021 Society of Photo-Optical Instrumentation Engineers (SPIE) [DOI: 10.1117/1 .JATIS.7.3.035009]
\end{abstract}

Keywords: vacuum ultraviolet; sounding rocket; radiometric calibration; sun-as-star.

Paper 21013AS received Jan. 25, 2021; accepted for publication Sep. 3, 2021; published online Sep. 27, 2021.

\section{Introduction}

Full-sun Ultraviolet Rocket SpecTrograph (FURST) is a sounding rocket instrument that will capture the first high-resolution, full-disk, and extreme ultraviolet (EUV) solar spectrum. It will measure the solar spectrum from 120 to $180 \mathrm{~nm}$ with a resolving power $>2 \times 10^{4} \lambda / \Delta \lambda$. The instrument's suborbital flight is currently scheduled for launch in 2022 and will capture $\sim 5 \mathrm{~min}$ of science data. The resulting solar spectra will be comparable in range and resolution to stellar spectra produced by the Hubble Space Telescope (HST) Space Telescope Imaging Spectrometer (STIS). To produce perfectly analogous data products, an absolute radiometric calibration of 5\% is required. However, because this is a highly ambitious goal, for the initial flight of the FURST instrument, we set our requirement on absolute radiometric calibration to $15 \%$. Calibration goals

*Address all correspondence to Genevieve D. Vigil, genevieve.d.vigil@nasa.gov 
and requirements are explained in detail in Sec. 3.1. Using a spectrally calibrated hollow cathode (HC) UV source, similar to that used for the calibration of the HST, the spectral calibration goal, of $\pm 3 \mathrm{~km} / \mathrm{s}$, can be readily achieved assuming adequate SNR is achieved in spectral line measurements. Absolute radiometric calibration of 5\%, however, is difficult to accomplish since a solar sounding rocket instrument is less capable of using stellar targets with well understood spectral emissions as references during a short daytime flight as is performed for HST. ${ }^{1,2}$ High quality absolute radiometric calibration can be achieved using the National Institute for Standards and Technology (NIST) Synchrotron Ultraviolet Radiation Facility (SURF) because the synchrotron radiation is a well studied and understood source, capable of calibration of $<1 \%$ in the FURST spectral range. However, due to size and material restrictions inherent to the SURF vacuum chamber, which are incompatible with initial designs of the FURST instrument, such calibration plans have been revised. Altered calibration plans now include the use of a portable calibration system to perform both the radiometric and wavelength calibration necessary for the FURST instrument. Using such a system, though the radiometric calibration goals of $5 \%$ is not achievable, this work estimates calibration at the level $\leq 6 \%$ can be realized for the FURST spectrum $\geq 134 \mathrm{~nm}$ and $\leq 12 \%$ for the range between 120 and $134 \mathrm{~nm}$. These calibration limits are primarily driven by the performance of NIST calibrated photodiodes (PDs) and fluctuations in the FURST instrument camera gain. In this study, the FURST instrument is briefly described in Sec. 2. The science objectives that drive the calibration system design are discussed in Sec. 3. The design of the portable calibration system is described in Sec. 4, and the methods that are to be employed to perform calibration of the FURST instrument are summarized in Sec. 5. Finally, a summary of the progress of FURST calibration and future work is given in Sec. 6.

\section{FURST Instrument}

The FURST instrument will capture high-resolution, UV spectra of the integrated solar-disk without a slit or a scanning mechanism. As the optical design for the FURST instrument was frozen only during the preparation of the present paper, and the instrument paper is still in preparation, this section provides a brief summary of the instrument design. The layout of the instrument is shown in Fig. 1, and Table 1 summarizes the design parameters. FURST employs a modified Rowland circle mount. The classic Rowland circle spectrometer has a fixed feed point

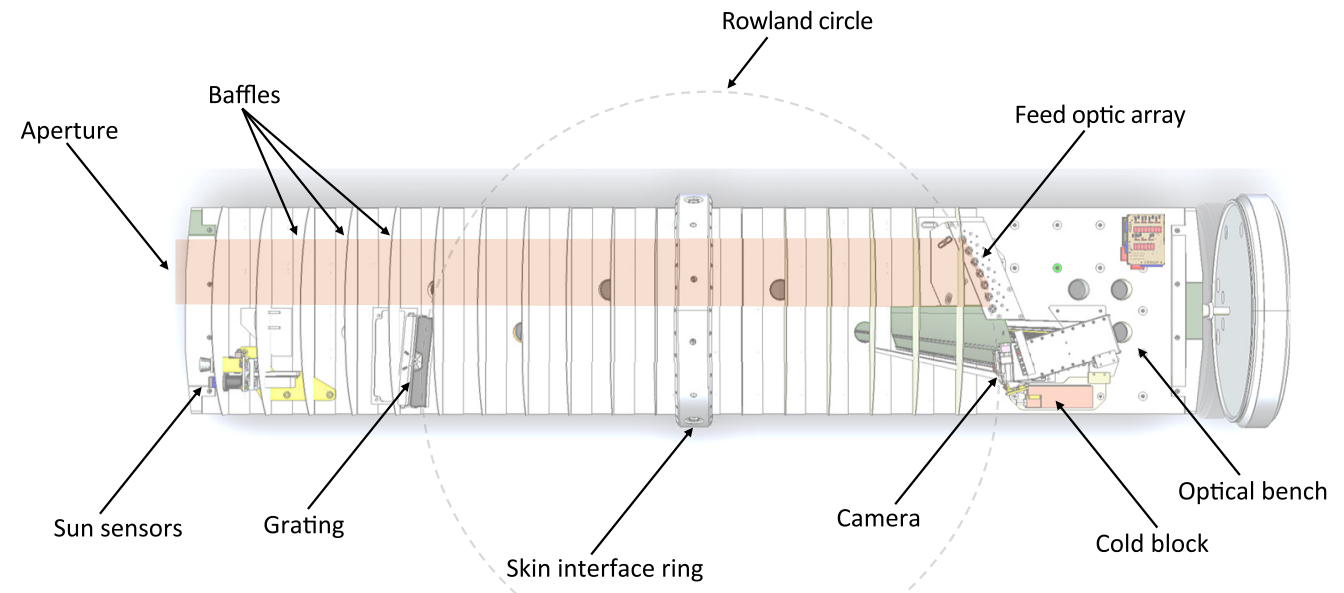

Fig. 1 Layout of the FURST instrument. Light enters the instrument from a rectangular aperture on the left and is collected and dispersed by one cylindrical feed optic at a time. The array of feed optics are positioned around the Rowland circle. The grating diffracts the light from the illuminated feed optic, focusing the spectrum onto the camera. Baffles and a visible-blind filter (on the camera head, not shown) are used to control stray light. 
Table 1 FURST design characteristics and (below the horizontal rule) expected performance.

\begin{tabular}{lc}
\hline \hline Grating radius & $1.35 \mathrm{~m}$, concave \\
Ruling pattern & $2200 \mathrm{gr} / \mathrm{mm}$, uniform \\
Feed optic radius & $3 \mathrm{~mm}$ \\
Feed optic positions & $18 \mathrm{deg}$ from grating normal \\
Number of channels & 7 \\
Detector position & 5 deg from grating normal \\
CCD format & $2048 \times 1024$ frame transfer \\
Pixel size & $15 \mu \mathrm{m}$ \\
Camera gain & $1 e^{-} / D N$ \\
Geometric area & $1.54 \times 10^{-6} \mathrm{~m}^{2}$ \\
Effective area & $1.02 \times 10^{-8} \mathrm{~m}^{2}$ \\
Spectral sampling interval & $0.005 \mathrm{~nm}$ \\
Single channel spectral range & $120 \mathrm{ton} 183 \mathrm{~nm}$ \\
Effective resolving power & $10 \mathrm{~nm}$, with $\sim 10 \%$ overlap \\
Full spectral range & $\geq 2 \times 10^{4} \lambda / \Delta \lambda$ \\
\hline \hline
\end{tabular}

on the grating axis and a movable detector. FURST instead has a fixed detector and an array of feed points, which allow the instrument to cover a spectral range from 120 to $183 \mathrm{~nm}$. The feed point and detector are both off-axis, on the same side of the grating normal, and placed close to the Littrow angle. This arrangement allows our large $(\sim 1.5 \mathrm{~m}$ diameter $)$ Rowland circle spectrometer to fit within a relatively narrow $(\sim 0.5 \mathrm{~m})$ rocket payload. Each feed point is a convex cylindrical mirror of 3-mm radius, oriented parallel to the grating rulings. The feed optic forms a one-dimensional $\sim 15 \mu \mathrm{m}$ wide virtual solar image parallel to its axis, which in fold is imaged 1:1 onto the detector. The feed optic thereby takes the place of a spectrometer slit and ensures unbiased sampling of radiation from the full Sun. Broadband VUV reflective coatings are applied to the feed optics and the diffraction grating.

Rather than scanning a single feed optic, we use an array of seven feed optics to attain the full spectral range of the instrument. Each feed optic produces one segment of the full spectrum, which is referred to here as a channel. The spectral ranges of adjacent channels overlap slightly so that the data can be combined into an uninterrupted spectrum. Each feed optic is housed inside a cylindrical shutter, which is motorized to rotate and open or close when triggered. The spectrum is thus formed by seven exposures, sequentially shuttering through the array of feed optics.

Light from the feed optic is dispersed and focused by the grating onto a single low-noise CCD camera, which is cooled via a heat strap to a cold block cooled by liquid nitrogen. The CCD detector is clocked slightly so that the columns are tilted by a few milliradians with respect to the spectral line. This allows an effective sampling rate much higher than the pitch of the detector pixels and thereby allows the instrument to achieve resolution apparently in excess of the Nyquist limit for our detector-though, of course, the pixel width still contributes to the line spread function of the spectrometer. Carefully designed baffling and a visible-blind filter on the camera head are used to limit stray light.

The instrument will be enclosed within 22 inch diameter sounding rocket skins. The rocket skins act as the instrument vacuum chamber for ground testing. 


\section{Calibration Requirements}

FURST is a unique payload that will enable studies of the Sun that will contribute to answering questions about the origins of solar activity, the nature of interactions between the sun and solar system and interstellar medium, and help characterize the fundamental processes that occur within the Heliosphere and throughout the universe. The experiment represents a solar-stellar missing link, which enables the sun to be studied as a star and directly compared to astrophysical measurements. By producing high quality VUV data, FURST will also provide insights to chromospheric and coronal heating.

\subsection{FURST Science Objectives}

FURST has numerous long- and short-term scientific goals. Though the initial sounding rocket flight will focus on solar heating observations, a future FURST-like satellite mission may enable a wide range of scientific observations, therefore the instrument is designed with all such goals in mind. To enable sun-as-a-star, heliospheric and solar system studies, FURST must measure the UV spectral irradiance of the sun. To reliably compare data products with HST, FURST data must be calibrated radiometrically and be of sufficient resolution. A spectral resolution of $\geq 2 \times$ $10^{4} \lambda / \Delta \lambda$ enables comparable analysis to HST STIS and the ability to resolve non-Gaussian lines core profiles seen in sun-like stars. Absolute radiometric calibration of FURST should be as close to HST calibration of 5\% as possible. To discern the small scale details expected of magnetic reconnection and dissipation (explosive events and microflares), FURST must achieve an SNR of $\geq 20$ to distinguish broad features from the continuum. Some other interesting science that FURST-like instruments will enable is the study of solar system bodies, such as the resonant absorption in the atmosphere of Europa, which requires an absolute wavelength calibration of $\pm 3 \mathrm{~km}$. FURST can also be used to quantify the $\mathrm{L} \alpha$-solar system interactions and thus needs a dynamic range capable of measuring unsaturated $\mathrm{H} \mathrm{L} \alpha$ signals, which can be $\approx 100 \times$ brighter than other lines. Finally, to capture a number of plasma diagnostic lines, FURST covers the spectral range from 120 to $180 \mathrm{~nm}$. Even though the initial flight of FURST will not be able to answer these science questions, all of the science goals discussed above are drivers of the calibration requirements, which this work is concerned about.

\subsection{Calibration System Requirements}

The above scientific observations provide the measurement requirements for the FURST instrument and therefore flow down to what is needed from the calibration system. The two calibration related goals that impact the design of the calibration system are (1) absolute wavelength calibration $\leq \pm 3 \mathrm{~km}$ over the entire FURST spectral range and (2) absolute radiometric calibration close to the HST level of $\approx 5 \%$ with in-channel relative calibration of $\approx 1 \%$. The first calibration goal is likely to be achievable because well calibrated spectral sources exist, such as elemental HC lamps emitting numerous well characterized spectral lines, e.g., of an excited Platinum source in a Nitrogen filled lamp. ${ }^{3}$ Measurement of $\approx 10$ sufficiently bright and well-resolved spectral lines in each channel is adequate to achieve the absolute spectral calibration requirements because sources of this type have been well studied and have spectrum known to $\leq 0.001 \AA$. However, such sources have not been as rigorously studied for absolute radiometric emission, largely due to the lack of absolute calibrated PDs in this spectral range and the absence of a full model of the complicated spectral emission of elemental HC sources. Synchrotron emission can provide the absolute radiometric calibration required to characterize the FURST system, such as that available at the NIST SURF III facility. However, the current design of the FURST instrument is not capable of fitting inside the NIST SURF III vacuum chamber without serious redesign and the material of the FURST optical bench is highly porous and unsuitable for the high vacuum requirements of the NIST facilities. Specifically, NIST has strict requirements about novel materials in their vacuum chambers since what would be considered minute levels of molecular contamination for a rocket mission can interfere with NIST?s long term operational requirements. Concerns were raised that the porous optical bench material would require excessive pumping times to achieve the high vacuum needed for safe operation with the Synchrotron

J. Astron. Telesc. Instrum. Syst. $\quad$ 035009-4 Jul-Sep 2021 • Vol. 7(3) 
Vigil et al.: Design for a portable calibration system for the Full-sun UV Rocket SpecTrometer instrument

Table 2 Calibration system requirements.

\begin{tabular}{lc}
\hline \hline Full spectral range & 120 to $180 \mathrm{~nm}$ \\
Absolute radiometric calibration & $15 \%$ over full range \\
Relative radiometric calibration & $5 \%$ over $50 \mathrm{px}$ range \\
Wavelength calibration & $\pm 3 \mathrm{~km}$ \\
Calculated line intensity & $1.0 \times 10^{4} \mathrm{e}^{-}$ \\
Spectral lines/channel & 10 \\
Line reference & $\leq 0.01 \AA$ \\
Alignment & $1 \mathrm{mrad}$ \\
Source angular size & $\leq 900^{\prime \prime}$ \\
Beam size & $7.45 \times 1.69 \mathrm{in}$ \\
Beam variability & $\leq 1 \% \mathrm{in}$ \\
Chamber pressure & $\leq 0.1 \mathrm{mTorr}$ \\
Test time & $\leq 5 \mathrm{~h}$ \\
\hline \hline
\end{tabular}

facilities. However, even without the ambitious radiometric calibration of 5\%, the FURST experiment provides a unique and important data set and therefore an alternative calibration requirement is proposed for this initial iteration of FURST. We set the FURST radiometric calibration requirement to a level that can be achieved through the use of NIST calibrated PD transfer standards. Using these calibrated PDs in conjunction with a wavelength calibrated Pt HC lamp, absolute radiometric calibration of $\leq 15 \%$ is expected (Table 2).

In addition to the requirements stated above, the calibration system is designed to achieve several other goals. The system will travel with the instrument to perform testing at various locations, and therefore must be robust, portable, and provide reproducible illumination. It must cover the full range of the FURST passband with sufficient intensity to enable reasonable testing timelines. The beam must illuminate the entire FURST collection optic array simultaneously and must be aligned to the instrument optical axis to at least the same precision the instrument will be aligned to the Sun. It must interface directly with the FURST instrument skins and hold stable vacuum and temperature conditions over the duration of a test since the instrument will be mounted inside the rocket skins. The overall costs of such a system should be commensurate with sounding rocket missions, the fundamental design should be flexible to support future mission calibration needs.

\section{Calibration System}

To achieve the calibration requirements outlined in the previous section, a portable calibration unit consisting of a Pt HC lamp, monochromoter, vacuum chamber, collimator, and calibrated PDs will be interfaced to the FURST sounding rocket instrument. This system will present to the instrument an approximate sun-sized source with pre-characterized irradiance and spectrum. Wavelength calibration can be performed with greater fidelity without a monochromator. However, radiometric calibration will occur by scanning a few several bright lines in each channel and comparing measurements acquired at the PD and instrument camera.

The calibration system is housed in a stainless steel vacuum chamber. The chamber has several ports in which to interface pumping hardware, input light, PD electronics, vacuum, and contamination monitoring hardware. The chamber has a large door at the back of the optical system, opposite the beam exit port, in which alignment and manual manipulation of internal hardware can be performed in air. The optical system is constructed atop an un-anodized 
aluminum optical bench mounted from the bottom of the chamber. The collimator system consists of an $f / 4.5$ Newtonian telescope composed of a parabolic primary and a flat, rectangular fold mirror. All optics are coated with Acton \# 1200 broadband UV coating providing $\approx 80 \%$ reflectively from 120 to $180 \mathrm{~nm}$. The mirrors are mounted on tip/tilt and $x / y / z$ stages for fine alignment adjustments and optical alignment elements are placed in the chamber and mounted to the primary mirror to perform high quality alignment. The collimator interfaces with a filter wheel and monochromator in series. The lamp is mated to the monochromator with a lamp housing containing a focusing mirror to direct and focus the light onto the entrance slit of the monochromator. The lamp is a platinum HC source with a custom magnesium fluoride window to pass the spectrum of interest similar to that used for HST calibration and studied. ${ }^{4,5,6}$ The spectral emission from such sources is known to $0.001 \AA$ thereby providing the key spectral calibration standard. In addition to vacuum pumps and pressure measurement sensors, a residual gas analyzer will be installed onto the chamber to monitor contaminant gases, such as water vapor, which absorb strongly at $L \alpha$. Two NIST calibrated Si PDs are used as transfer standards to quantify the irradiance at each wavelength before entering the FURST instrument. Two PDs are required to limit ambiguities that arise from temporal fluctuations during a measurement sequence, as well as potential contamination issues. The PDs will be calibrated before and after flight at NIST to quantify any potential degradation. The NIST transfer standard are the core of radiometric calibration and as such are a critical subsystem to the calibration design. The entire chamber and supporting vacuum and electronic support hardware are mounted on a portable cart, which can be raised and lowered to mate directly to the rocket skins of the FURST instrument. Figure 2 shows a diagram of the calibration system design.

\subsection{Simulated Calibration Data}

The signal levels of various emission lines can be estimated to better design a calibration system capable of meeting the above-mentioned requirements. We combine the Pt HC irradiance measurements reported in Ref. 6 and the Pt atlas studied ${ }^{5}$ to generate a full spectrum from 120 to $180 \mathrm{~nm}$ with approximate irradiance. The signal at the PD and instrument camera then can be estimated by accounting for attenuations and conversions expected in the system. Presence of

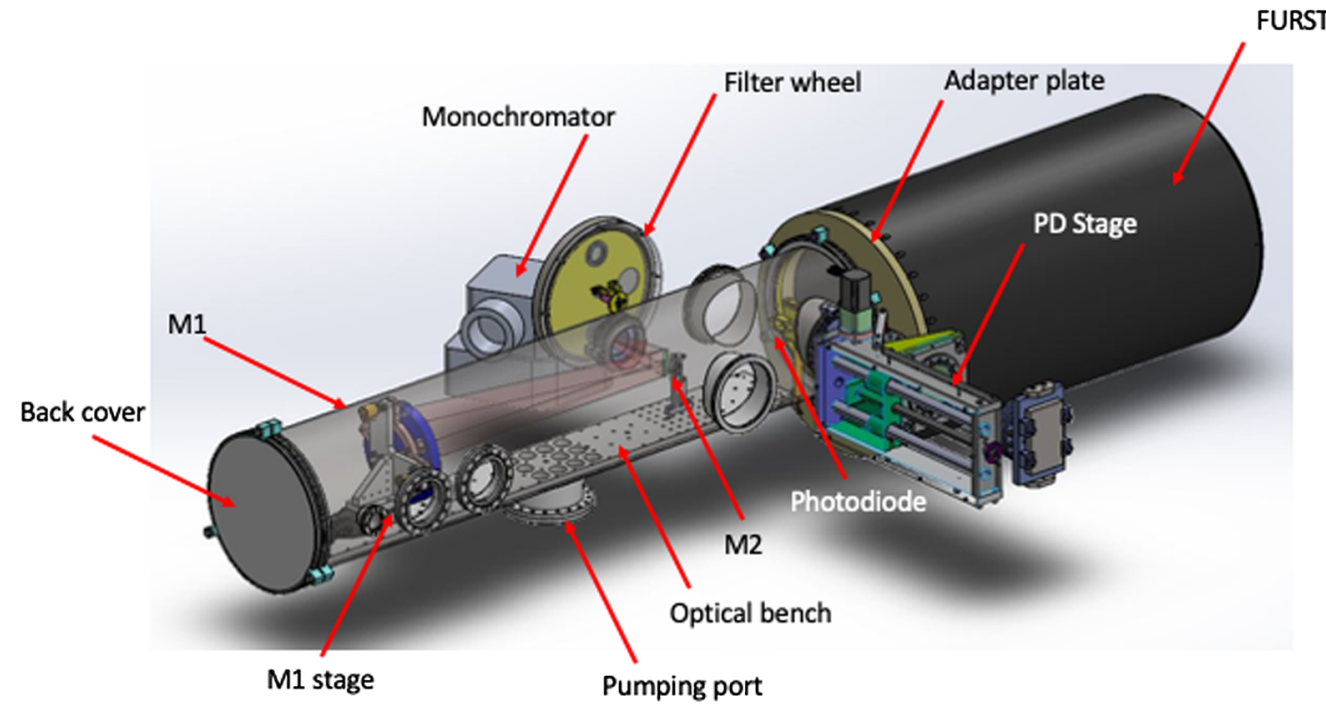

Fig. 2 Optical layout of the integrated FURST instrument and calibration system. The calibration system consists of a simple on-axis telescope with a $f / 4.5$ parabolic primary (M1) and a rectangular fold mirror (M2) coupled to a monochromator and HC lamp (not shown). The entire optical assembly is housed inside of a cylindrical vacuum chamber and laid out on an optical bench. The main calibration PD is mounted on a $2 \mathrm{D}$ stage, which can translate and sample the entire collimated beam entering the instrument. A broad band UV filter will be placed near the monochromator exit slit to minimize scattering. An adapter plate will be used to mate the collimator chamber to the FURST rocket skins. 
absorbing gases such as water and $\mathrm{O}_{2}$, throughput of optical elements, responsivity, and noise performance of the PDs can all be accounted for to generate expected signal strength of any particular spectral line. below.

The signal measured at the calibration system PD can be estimated according the relationship

$$
S_{p d}(\lambda)=I_{c}(\lambda) A_{p d} R_{p d}(\lambda) \frac{h c}{\lambda}
$$

where $I_{c}$ is the irradiance at the exit port of the collimator in $\mathrm{ph} / \mathrm{s}-\mathrm{cm}^{2}, A_{p d}$ is the area of the PD in $\mathrm{cm}, R_{p d}$ is the responsivity of the PD in $A / W, h$ is the Plank's constant in $J-s, c$ is the speed of light in $\mathrm{m} / \mathrm{s}$, and $\lambda$ is the wavelength in $m$. The wavelength dependent irradiance can be written as

$$
I_{c}(\lambda)=\frac{I_{P t}(\lambda) \gamma_{c}(\lambda) \Omega d A T_{a b s, c}(\lambda)}{A_{\text {beam }}},
$$

where $I_{P t}$ is the spectral irradiance of the HC Platinum source in $\mathrm{W} / \mathrm{cm}^{2}$-sr. $\gamma_{c}$ is the unitless throughput of all optical elements including monochromator slit and reflective surfaces. $d A$ is the area of the emitting element in $\mathrm{cm}$ and is related to $\Omega$, the solid angle the HC source emits into in sr, by $\Omega=d A /\left(4 \pi f^{2}\right) . f$ is the focal length of the collimator. $T_{\text {abs }}=\exp \left[-k_{x x} l_{c} P P_{x x}\right]$ is the absorption due to contaminating gasses such as water vapor and $\mathrm{O}_{2}$, where $l_{c}$ is the path length in $\mathrm{cm}, k_{x x}$ is the absorption coefficient in $\mathrm{cm}^{-1}$, and $P_{x x}$ is the ratio of the partial pressure of the contaminant to the total pressure. ${ }^{7}$

The signal at the instrument camera can be computed according to

$$
S_{i}(\lambda)=\frac{I_{c}(\lambda) A_{\mathrm{eff}} Q E \eta G t T_{i}}{N_{p x}},
$$

where $A_{\text {eff }}$ is the effective area of the instrument. $Q E$ is the Quantum efficiency of the camera. $\eta=\frac{h c}{\lambda} \frac{1}{E e h}$ is the quantum yield, where $E_{e h}$ is the electron generation energy in $\mathrm{Si}$ of $3.65 \mathrm{eV} / \mathrm{e}^{-}$. $G$ is the gain in $\mathrm{e}^{-} / \mathrm{DN}$ and $t$ is the integration time in $s . T_{i}$ is the attenuation due to the presence of absorbing contaminant gases in the instrument $T_{i}=\exp \left[-k_{x x} l_{i} P P_{x x}\right]$, where $l_{c}$ is the instrument path length. $N_{p x}$ is the number of pixels the signal is spread over.

The ultimate goal of the calibration measurement is to quantify the effective area, combined with the quantum efficiency, of the instrument with errors $\leq 15 \%$. This can be achieved by taking the ratios of Eqs. (3) and (1)

$$
A_{\text {eff }} Q E=\frac{S_{i} N_{p x} A_{p d} R_{p d} \frac{h c}{\lambda}}{S_{p d} T_{i} G t \eta}
$$

The effective area of the FURST instrument will be quantified at spectral locations at which the PD transfer standard has been calibrated. Therefore, $\sim 2$ radiometric measurements centered at bright lines in each channel, for a total of no more than 14 measurements, will adequately sample the full spectrum and yield the radiometric calibration.

We can estimate the signal magnitudes at the PD and the instrument camera by mapping the full $\mathrm{Pt}$ atlas ${ }^{5}$ in relative intensity units to absolute units approximated by the measurement of a few bright lines. ${ }^{6}$ We can then account for absorption, reflections, and PD sensitivities to estimate the signal measured at the photoidode. The spectral variation of PD responsivity, attenuation coefficients due to water vapor and molecular oxygen and optical coating reflectivity are given in Fig. 3. Any additional values required to estimate signal levels are reported in Table 3.

Starting with the spectral irradiance plotted in Fig. 3, estimated signal for $S_{i}(\lambda)$ can be calculated. We simulate imaging data by summing 10 frames of $100 \mathrm{~s}$ exposures having average noise of $30 e^{-}$per exposure, based on previous measured performance of an identical camera originally designed for the EUV Snapshot Imaging Spectrograph (ESIS, publication in process) mission, camera performance operated at $-70^{\circ} \mathrm{C}$. An example of the simulated spectral data is 
Vigil et al.: Design for a portable calibration system for the Full-sun UV Rocket SpecTrometer instrument
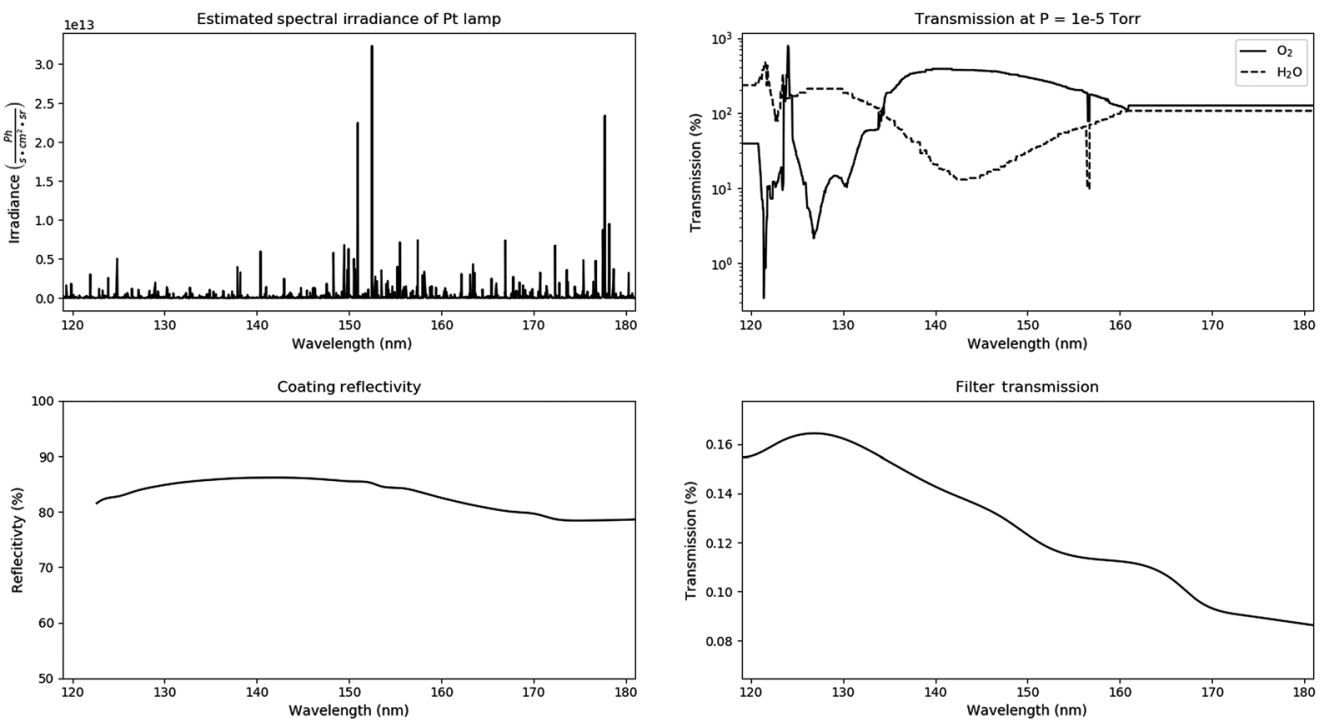

Fig. 3 Expected spectral behavior of calibration system factors. Pt lamp irradiance is estimated from. ${ }^{5}$ Absorption coefficients for water vapor and oxygen are taken from. ${ }^{7}$ Coating reflectivity used on all four optical surfaces in the calibration system, Acton \# 1200, is given in Ref. 8. Calibration PD spectral responsivity is estimated from NIST reports at Ref. 9.

Table 3 Simulation data values.

\begin{tabular}{lccc}
\hline \hline Variable & Value & Budgeted error (\%) & Expected error \\
\hline$A_{\text {eff }}$ & $1.02 \times 10^{-8} \mathrm{~m}^{2}$ & $\mathrm{NA}$ & - \\
$Q E$ & 0.5 & $\mathrm{NA}$ & - \\
$G$ & $1 e^{-} / D N$ & 3 & $1 \%$ \\
$t$ & $100 \mathrm{~s}$ & 1 & $1 e-3 \mathrm{~s}$ \\
$I_{c}$ & $2 \mathrm{~m} \mathrm{~s}$ & 1 & $1 \mathrm{~mm}$ \\
$I_{i}$ & $4.5 \mathrm{~m} \mathrm{~s}$ & 1 & $1 \mathrm{~mm}$ \\
$d A$ & $3 \mathrm{~mm} \mathrm{~m}^{2}$ & 1 & $1 \%$ \\
$A_{\text {beam }}$ & $\pi 4.5^{2}$ in & 1 & $0.1 \mathrm{in}$ \\
$N_{p x}$ & $1024 \mathrm{px}$ & 1 & $5 \mathrm{px}$ \\
$P_{x} X$ & $\frac{1 \times 10^{-4}}{760}$ Torr & 1 & $1 \times 10^{-5}$ \\
$R_{p d}$ & Fig. 3 & 11 & $6 \%$ to $11 \%$ \\
$S_{p d}$ & Fig. 5 & 5 & $1 \%$ to $5 \%$ \\
$S_{i}$ & Fig. 4 & 8 & $1 \%$ to $8 \%$ \\
\hline \hline
\end{tabular}

given in Fig. 4. An example image of the expected results from FURST channel 1, a region of the Pt spectrum containing few bright lines, is shown along with the quality of a radiometric measurement. Each measurement is taken as the sum along the row of pixels illuminated by light that passes through a slit centered at $1246.8 \pm 5 \AA$. Each row is treated as an independent measurement and the statistics along with a Gaussian fit and error estimate are given in Fig. 4. This measurement is combined with the measurement at the calibration system PD, shown in 

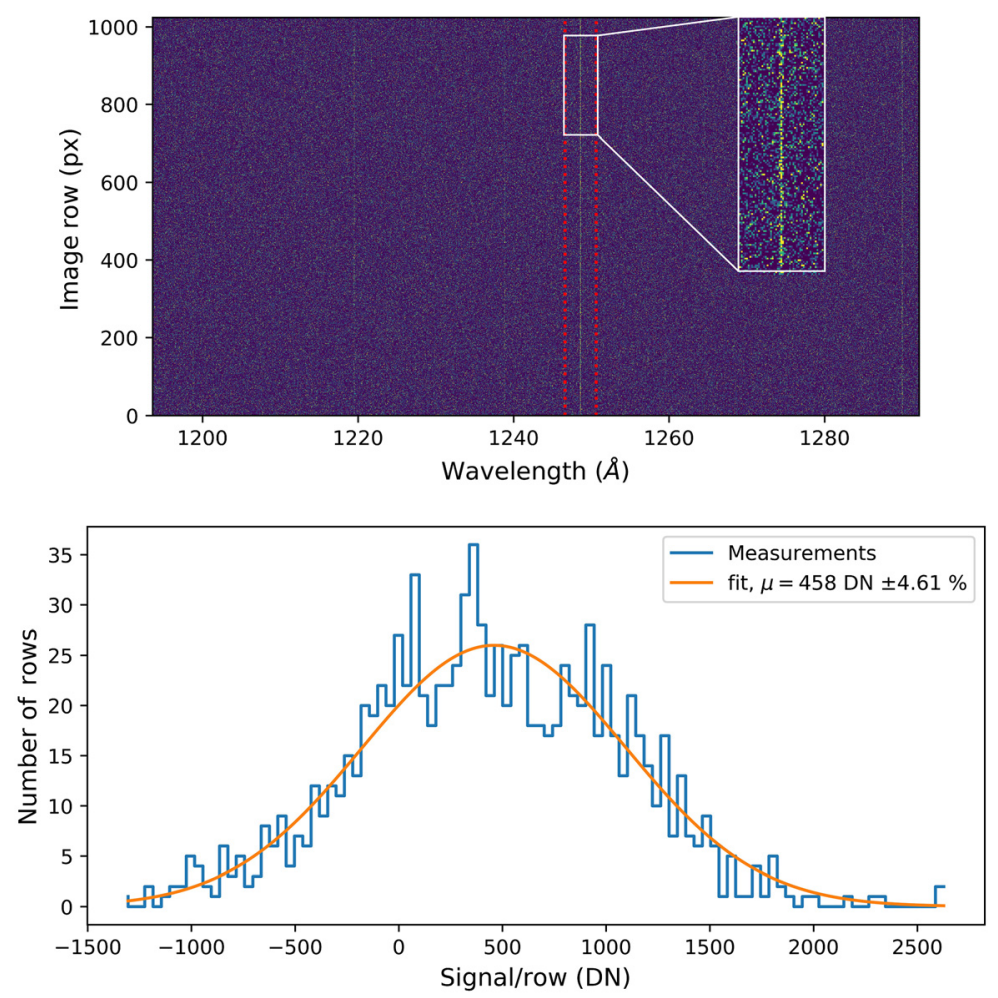

Fig. 4 Simulated FURST calibration data representative of the spectral range covered by channel 1 of the FURST instrument. (a) The imaging data represents the sum of 10 exposures of $100 \mathrm{~s}$ each. A The inset shows a zoomed in region around the spectral line of interest to better display the expected relative brightness of the signal to background. Between red dashed lines represents the slit passband centered at $1248.6 \pm 5 \AA$. (b) Each row is summed horizontally in the slit passband and treated as an individual intensity measurement for which the statistics are histogrammed and fitted to estimate measurement uncertainty. In this case, the result of performing this analysis of the 1248.6 line yields an intensity measurement in DN within budgeted error.

Fig. 5, to quantify the true Effective Area of the instrument according to Eq. (4). The example PD measurement in Fig. 5 is computed using the expected photon flux rates given, a slit centered at $1246.8 \pm 5 \AA$, shot noise, a 50 s collection time, sampling at a 10 samples/s rate, and digitized according to a Keithly 6485 picoameter. Exposure times are tuned such that both measurements meet the error budgets listed in Table 3 at the estimated noise levels.

The radiometric calibration uncertainty is expected to be dominated by PD responsivity, PD and camera measurement uncertainty, and gain stability. The gain of this camera design was quantified for the ESIS mission, to vary somewhat with temperature and we plan to quantify gain uncertainty in near real time both during calibration tests and flight using an Fe-55 source (more discussion in Sec. 5.2.1). We perform error propagation to estimate the overall expected calibration uncertainty by simulating the signal measured at each detector and computing Eq. (4) using values given in Table 3 . The resulting spectrally varying measurement of effective area can be obtained as shown in Fig. 6 and shows that given the error budget presented here, the $\leq 15 \%$ radiometric error is achieved for the entire FURST spectrum.

\section{Calibration Methods}

Once the calibration system is fully developed and tested, it will be transported to Montana State University (MSU) facilities, where it can be integrated with the FURST instrument. Additionally, the calibration system will be transported with FURST to the launch site where final pre-launch and post-launch characterization will be performed. 
Vigil et al.: Design for a portable calibration system for the Full-sun UV Rocket SpecTrometer instrument

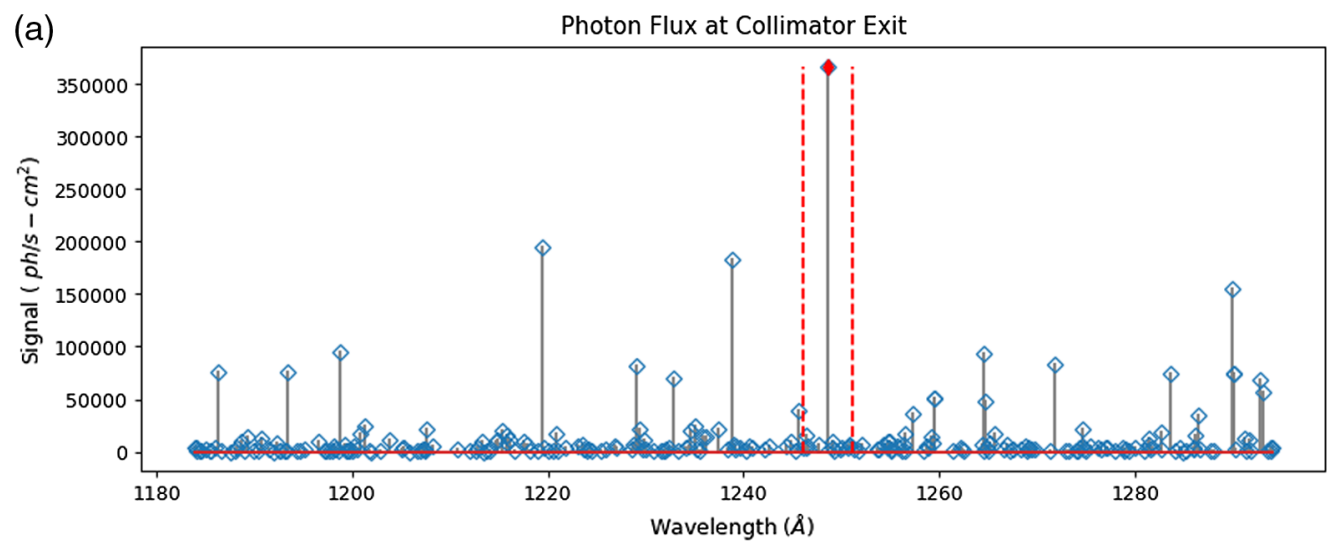

(b)

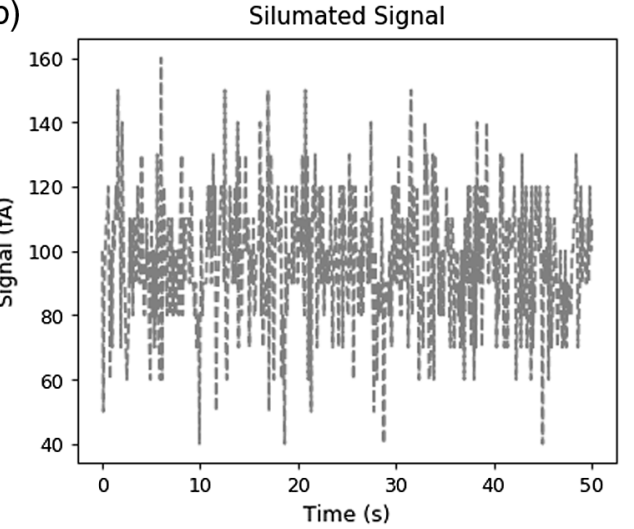

(c)

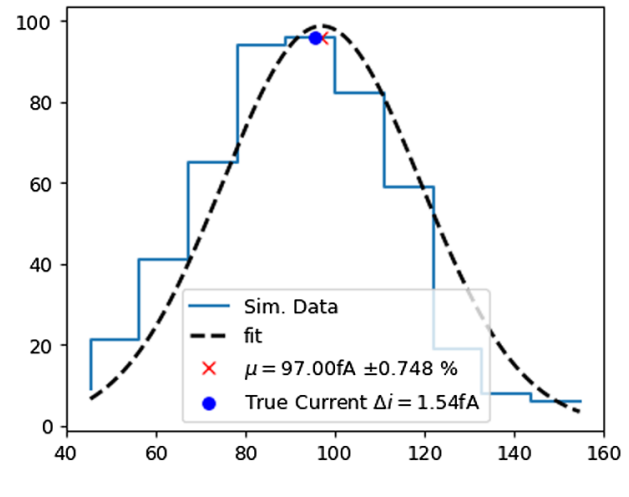

Fig. 5 (a) Example of simulated PD data and measurement error. Estimated photon flux at the exit of the collimator in channel 1. The slit bandpass, centered at $1248.6 \pm 5 \AA$, is noted with red dashed lines. (b) Simulated PD signal- acquiring data for $50 \mathrm{~s}$ at 10 samples/s sampling rate is shown. (c) Histogram of simulated data with Gaussian fit showing measurement error well within the error budget for PD measurements of $\leq 5 \%$ is shown.

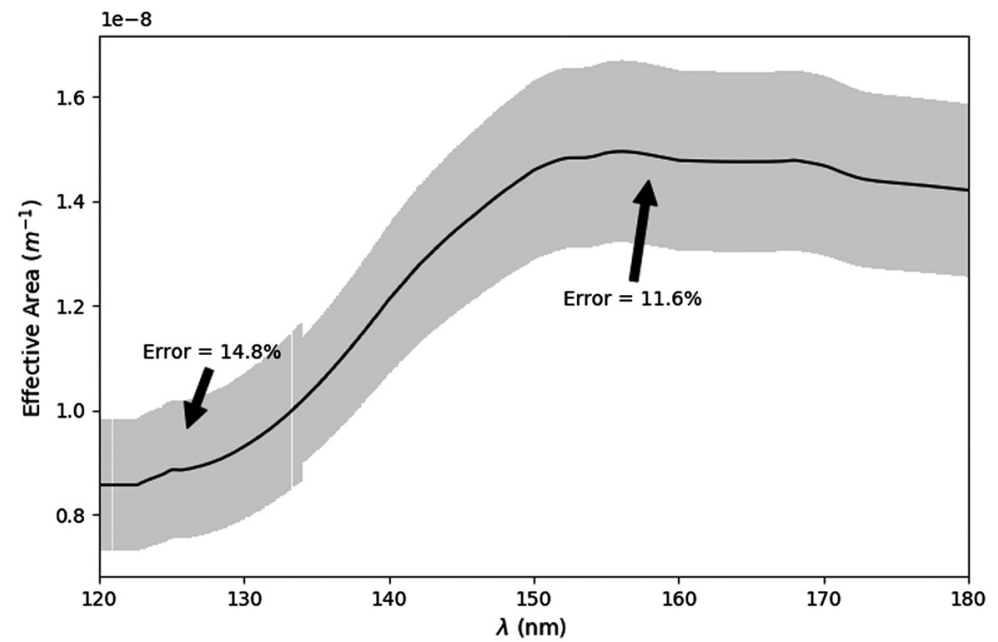

Fig. 6 Calculated effective area and estimates of calibration error. Error is dominated by PD responsivity error, camera measurement error, PD measurement error, and gain error. Overall error is expected meet the $15 \%$ error requirement. 


\subsection{Radiometric Calibration Process}

Radiometric calibration requires the use of a monochromator to limit the spectral measurement to close to a single bright line. Once the location of bright lines has been established using a wide slit, a narrow slit width will be used and sequential measurements will be taken between the camera and the PD at the location in the beam that would expose the feed optic corresponding to the appropriate channel. Therefore the PD will be moved in and out of position between each measurement. Temporal variations will be accounted for with a second PD that will be acquiring data while the instrument is taking a measurement.

Because the spectral lines vary greatly in intensity, different integration times can be used to optimize the data collection process. Assuming 14 total radiometric measurements, at 30 to $60 \mathrm{~s}$ for each PD measurement, and 300 to $1000 \mathrm{~s}$ total, broken into $100 \mathrm{~s}$ exposures, for each instrument measurement, we estimate radiometric measurements can be completed in 1.5 to $4.5 \mathrm{~h}$ including sometime between each measurement type. Exposure times of $100 \mathrm{~s}$ exposures are chosen as a trade off between improved noise performance and the limit at which significant pile-up is expected in the gain measurements (Sec. 5.2.1). This estimated data collection time is easily achieved in a single day and can be performed before and after every significant event that has the potential to disturb instrument function, i.e., shipping or vibe testing.

\subsection{Wavelength Calibration Process}

Wavelength calibration will be done separately and is optimized by removing the monochromator from the calibration set up since the monochromator is responsible for significant throughput losses and is not needed for this calibration step. While wavelength calibration is not the primary focus of this work, it has been estimated that wavelength calibration can also be achieved in $\sim 1$ to $3 \mathrm{~h}$ total. Radiometric calibration and wavelength calibration are not expected to be fully performed on the same day. However, the slit width for radiometric calibration can be set wide enough to allow wavelength position to be checked between the two tests.

\subsubsection{Camera calibration}

As mentioned in Sec. 4.1, one of the potential sources of error that drive the absolute radiometric calibration quality is camera gain fluctuations. Previous iterations of the FURST camera for other sounding rocket instruments have shown the gain is subject to temporal fluctuations on the order of $1 \%$ to $3 \%$ depending on temperature as well as on other not yet determined factors. The gain varies on time scales of 1 to $10 \mathrm{~s}$ of seconds and gain drift from mean over longer periods, e.g., hours needed for calibration testing, is not known. Therefore, to establish absolute radiometric calibration that is valid over a $5 \mathrm{~min}$ flight or a $4 \mathrm{~h}$ calibration test, simultaneous gain quantification is needed. With adequate measurement statistics, we can reduce the overall gain uncertainty to eliminate it as a major source of calibration error. This can be achieved through in situ Fe-55 calibration in which the CCD is exposed to sufficiently sparse x-ray photon flux to measure numerous single photon events of known energy. We intend to expose a small section of each quadrant of the CCD to Fe-55 and use them as gain calibration pixels while the rest of the CCD is to be masked off from exposure to the source to limit the impact on science data. The Fe-55 hits can be separated from spectral data by energy, whereas the bulk of the science data is retained without interference from Fe-55 photons. We must balance the flux of the source between obtaining enough single pixel events to reliably compute gain while avoiding pile-up, or multi-photon events, which complicate analysis. Both multi-pixel events, photons absorbed in more than a single pixel, and multi-photon events, pixels that are struck by more than one photon in an exposure period, complicate gain analysis and are therefore to be avoided. Figure 7 shows a proposed layout for the on-board gain calibration. The source must be placed on the sensor side of the filter because Fe-55 photons will not transmit through the focal plain filter. The source will be attenuated by a pinhole and masked to limit exposure only intended region.

Figure 7 also shows simulated data for the FURST back-illuminated CCD device, which we have performed to approximate the flux needed to achieve a reliable gain measurement. A simplified simulation, based off of work found in Refs. 10 and 11 , assumes a $15 \times 15 \mu$ m pixel with

J. Astron. Telesc. Instrum. Syst. $\quad$ 035009-11 Jul-Sep 2021 • Vol. 7(3) 

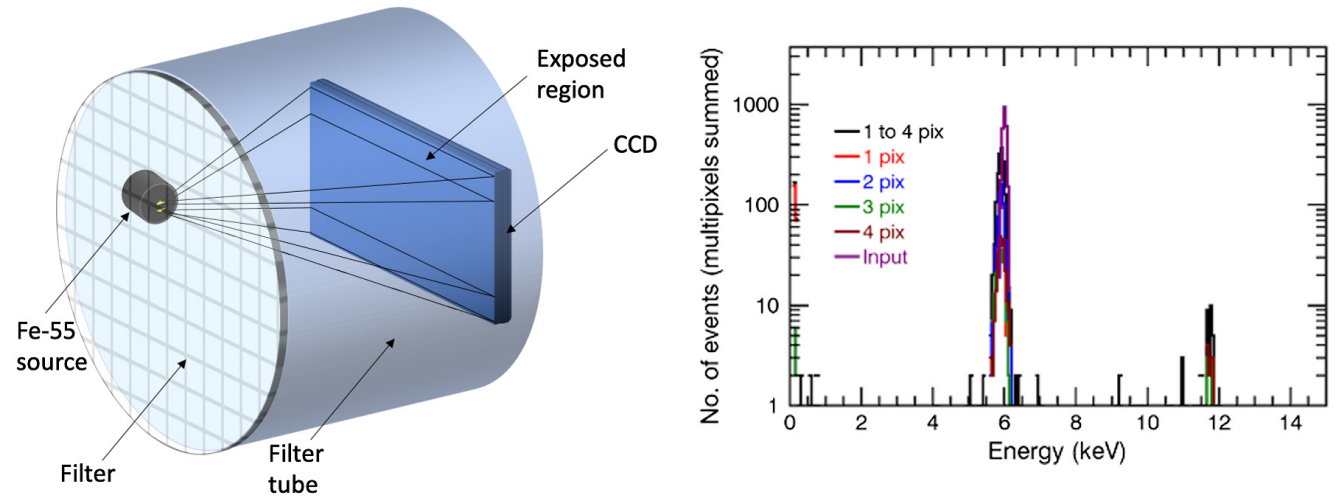

Fig. 7 (a) Simplified layout of in situ gain calibration. (b) Simulated Fe-55 exposure and event selection shows $>400$ single pixel, single photon, events occur out at $5.9 \mathrm{kEv}$ yielding a reliable gain measurement. Over $30 \%$ of all events are lost due to quantum efficiency in the CCD and $\sim 30 \%$ of the remaining events are single pixel hits seen in the red peak near $5.9 \mathrm{keV}$.

a $35 \mu \mathrm{m}$ depletion region and a total of 2500 photons distributed mainly in a single energy peak at $5.9 \mathrm{keV}$. Roughly $60 \%$ of all incident photons interact in the CCD and result in signal while $\sim 30 \%$ of the interacting photons are absorbed in a single pixel. This simulation informs the sizing and attenuation of Fe-55 source needed to obtain a confident gain measurement in every exposure.

\section{Discussion and Conclusions}

We have presented the design, operation, and expected performance of a portable VUV calibration system capable of performing simultaneous radiometric and wavelength calibration for the FURST sounding rocket. The absolute calibration of the FURST instrument to a comparable level to HST STIS, presents a significant challenge. While we do not expect to be able to achieve the exact same level of absolute radiometric calibration of the HST, we anticipate achieving absolute calibration $\leq 15 \%$. This level of calibration is sufficient for an initial flight of the instrument and serves as a basis for future calibration goals. The data products generated by FURST calibrated to this high level will represent the first measurement of the sun-as-a-star and can be directly used to shed light on solar heating mechanisms as well as compare as a snapshot to other stellar objects. We will achieve this calibration through the building of custom calibration ground support equipment, which is described in detail above. With this design, the absolute radiometric and wavelength calibration of FURST in the VUV can be performed simultaneously and without the need for synchrotron facilities. The system was designed with flexibility to adapt to future missions and provides MSFC with an important calibration facility available to develop high quality EUV instruments.

\section{Acknowledgments}

This work is funded by the Low Cost Access to Space in NASAs Heliophysics Technology and Instrument Development for Science program. Genevieve Vigil's research is partially supported by an appointment to the NASA Postdoctoral Program at the Marshall Space Flight Center, administrated by Universities Space Research Association under contract with NASA

\section{References}

1. R. C. Bohlin et al., "Spectrophotometric standards from the far-ultraviolet to the near-infrared: STIS and NICMOS fluxes," Astron J. 122, 2118-2128 (2001).

2. R. C. Bohlin, I. Hubeny, and T. Rauch, "New grids of pure-hydrogen White Dwarf NLTE model atmospheres and the HST/STIS flux calibration," Astron. J. 160(1), 21 (2020). 
Vigil et al.: Design for a portable calibration system for the Full-sun UV Rocket SpecTrometer instrument

3. N. Donders et al., "Wavelength calibration of the full-Sun ultraviolet rocket SpecTrometer (FURST)," in AGU Fall Meeting Abstr., p. SH31C-3325 (2019).

4. F. Kerber et al., "Spectral characterization of HST calibration lamps: new Pt/Cr-Ne line catalogues and aging test," Proc. SPIE 5488, 679 (2004).

5. J. E. Sansonetti et al., "Atlas of the spectrum of a platinum/neon hollow-cathode reference lamp in the region 1130-4330 A," J. Res. Natl. Inst. Standards Technol. 97(1), 1-211 (1992).

6. J. Z. Klose, G. F. Hartig, and W. J. Rosenberg, "Characterization of a Pt-Ne hollow cathode spectral line source," Appl. Opt. 29(19), 2951 (1990).

7. A. L. Buck, "me variable-path Lyman-alpha hygrometer and its operating characteristics," Bull. Am. Meteorol. Soc. 57, 1113-1119 (1976).

8. T. Acton, "Products: $\mathrm{Al}+\mathrm{MgF}_{2}$ broadband mirrors," actonoptics.com/products/al-mgf2mirrors.

9. R. Vest, "Si EUV photodiode transfer standard," https://www.nist.gov/laboratories/toolsinstruments/si-euv-photodiode-transfer-standard.

10. P. S. Athiray et al., "Simulating charge transport to understand the spectral response of Swept Charge Devices," Astron. Astrophys. 583, 1-8 (2015).

11. P. S. Athiray et al., "Calibration of the MaGIXS experiment I: calibration of the X-ray source at the X-ray and Cryogenic Facility (XRCF)," Astrophys. J. 905(1), 66 (2020).

Genevieve D. Vigil received her $\mathrm{PhD}$ in electrical engineering at the University of Notre Dame in 2017. She completed a post-doctoral fellowship at Marshall Space Flight Center on the Heliophysics Sounding Rocket team aiding in the development, testing and launch of various solar missions, including Hi-C 2.1, CLASP 2, and MaGIXS. Her research interests include novel solar instrumentation especially for EUV and X-ray solar applications as well as high resolution imaging and image processing techniques.

Biographies of the other authors are not available. 